\title{
REVIEW
}

Open Access

\section{Chest CT findings of COVID-19-infected patients, are there differences between pediatric and adult patients? A systematic review}

Javid Azadbakht ${ }^{1}$, Hamed Haghi-Aminjan ${ }^{2}$ and Bagher Farhood ${ }^{3^{*}}$ (D)

\begin{abstract}
Background: Purpose of this study was to deliver a report of chest CT findings of COVID-19-infected pediatric and adult patients and to make an age-based comparison. A systematic search was conducted in accordance with PRIS MA guidelines to identify relevant studies in the electronic databases of PubMed, Scopus, ProQuest, ScienceDirect, and Web of Sciences from January 1, 2020 to March 27, 2020 using search terms in the titles and abstracts. Based on our inclusion and exclusion criteria, 762 articles were screened. Finally, 15 eligible articles which had adequate data on chest CT findings of COVID-19-infected patients were enrolled in this systematic review.

Results: In pediatric patients (15 years old or younger), peripheral distribution was found in 100\% of cases, ground glass opacities (GGO) in 55.2\%, bilateral involvement in 50\%, halo sign in 50\%, unilateral involvement in 30\%, consolidation in $22.2 \%$, crazy paving pattern in $20 \%$, nodular opacities in $15 \%$, pleural effusion in $4.2 \%$, lymphadenopathy in none, and normal imaging in $20.8 \%$ of cases. On the other hand, in adult patients, bilateral involvement was reported in 76.8\%, GGO in 68.4\%, peripheral distribution in 62.2\%, mixed GGO and consolidation in $48.7 \%$, consolidation in $33.7 \%$, crazy paving pattern in $27.7 \%$, mixed central and peripheral distribution in $25.0 \%$, unilateral involvement in $15.2 \%$, nodular opacities in $9.2 \%$, pleural effusion in $5.5 \%$, central distribution of lesions in $5.4 \%$, lymphadenopathy in $2.4 \%$, and normal imaging in $9.8 \%$ of cases.

Conclusion: According to the findings of this systematic review, children infected with COVID-19 can present with normal or atypical findings (nodular opacities/unilateral involvement) in chest imaging more frequently than adult patients. Therefore, more caution should be taken to avoid misdiagnosis or missed diagnosis in infected children. Besides, clinical and laboratory findings need to be considered more decision-making for pediatric patients with normal or atypical chest CT scan but high suspicion of COVID-19.
\end{abstract}

Keywords: 2019 novel coronavirus, COVID-19, Chest CT, Thoracic CT, Lung, Pulmonary, Pediatric, Adult

\footnotetext{
* Correspondence: bffarhood@gmail.com

${ }^{3}$ Department of Medical Physics and Radiology, Faculty of Paramedical

Sciences, Kashan University of Medical Sciences, Kashan, Iran

Full list of author information is available at the end of the article
}

\section{Springer Open}

( ) The Author(s). 2020 Open Access This article is licensed under a Creative Commons Attribution 4.0 International License, which permits use, sharing, adaptation, distribution and reproduction in any medium or format, as long as you give appropriate credit to the original author(s) and the source, provide a link to the Creative Commons licence, and indicate if changes were made. The images or other third party material in this article are included in the article's Creative Commons licence, unless indicated otherwise in a credit line to the material. If material is not included in the article's Creative Commons licence and your intended use is not permitted by statutory regulation or exceeds the permitted use, you will need to obtain permission directly from the copyright holder. To view a copy of this licence, visit http://creativecommons.org/licenses/by/4.0/. 


\section{Background}

Coronaviruses are enveloped single-stranded RNA viruses which can rapidly mutate and recombinate and have basic reproductive number of about 2.2 [1]. Six coronavirus species can result in human disease [2]. Four species-229E, OC43, NL63, and HKU1-are prevalent which in immunocompetent individuals typically induce common cold symptoms [2]. The two other speciesSARS-CoV and MERS-CoV-are originally zoonotic and occasionally have been connected to fatal illness [3]. Due to high prevalence and wide distribution of coronaviruses, considerable genetic diversity and frequent genome recombination, and increasing activities which bring human and animal together, novel coronaviruses are possible to periodically infect humans because of frequent cross-species infections and occasional virus spillover [3, 4].

WHO (the World Health Organization) received data on some patients with unknown cause respiratory disease from Wuhan City, China, on December 31, 2019, with clinical presentations similar to those of viral pneumonia including symptoms of as fever, cough, and dyspnea. Also, patients often showed pulmonary opacities at chest imaging [5]. Bronchoalveolar lavage fluid sample analysis and electron microscopy showed coronavirus as causative infectant, a virus with a crown like morphology at electron microscopy resulted from viral spike peplomers coming out from the viral envelope [6]. This newly found strain has been temporarily named the 2019 novel coronavirus (2019-nCoV or COVID-19). The virus is highly contagious and can be transmitted from human to human by an infected person or an asymptomatic carrier and is capable of spreading rapidly between cities. Respiratory droplets are the main route of transmission, but close contact and transmission through digestive tract can play a role also [7]. Most of the cases are mild in clinical term, but the elderly or those with comorbidities are more likely to experience severe form of infection [8].

Humankind is generally susceptible to COVID-19 and no vaccination or definite treatment is at hand yet. Early detection and effective control of transmission pathways (i.e., isolation of suspected cases, disinfection, etc.) are still the most effective procedures to stop the COVID-19 outbreak. However, because of large number of clinically suspected COVID-19 patients, laboratory detection tests take a lot of time hindering early treatment and may not be feasible for all suspected patients owing to the low number of available COVID-19 test kits [9]. Moreover, due to the limitation of sampling materials in the early stage of the infection, the positive rate is relatively low [10]. These challenges increase spread chance by free movement of highly suspected cases who are not yet identified and quarantined.
Imaging plays a crucial role in the diagnosis and follow-up of novel coronavirus-infected pneumonia (NCIP) patients. CT is regarded as preferred imaging modality in clinically suspected cases and is valuable to monitor imaging changes during treatment. Therefore, CT has been considered as an effective diagnostic tool for clinically suspected COVID-19 [9]. It potentially can spot people with negative reverse transcription-polymerase chain reaction (RT-PCR) but yet highly suspicious of NCIP regarding clinical conditions $[10,11]$. CT scan findings may also provide information about the disease severity [12-15]. Furthermore, considering similarities in imaging patterns of most viral pneumonia, the CT features reported in many recent studies might be helpful to discriminate virus from other pathogens causing pneumonia and to triage patients.

Some studies have reported CT features of patients infected with COVID-19. However, chest CT findings of pediatric patients with COVID-19 infection may be different from adults. In this study, we aimed to review literature systematically to find differences in reported CT features in COVID-19-infected patients in pediatric and adult age group, considering major CT role in screening and triage of COVID-19 disease where there is no serological testing available or results are not returning in due time.

\section{Methods \\ Study protocol}

The present study was done according to the guidelines of Preferred Reporting Items for a Systematic Review and Meta-analysis (PRISMA) [16].

\section{Search strategy}

A systematic search was performed in electronic databases including PubMed, Scopus, ProQuest, ScienceDirect, and Web of Sciences from January 1, 2020 to March 27,2020 . The selected keywords were in accordance with our study purpose, "Diagnostic Imaging" OR "Diagnostic X-Ray" OR "Diagnostic X-Ray Radiology" OR "Medical Imaging" OR "X-Ray Computed Tomography" OR "CT" OR "X-Ray Computer Assisted Tomography" OR "CT X-Ray" OR "X-Ray CAT Scan" OR "X-Ray Computerized Tomography" AND "COVID-19" OR "2019-nCoV" OR "nCoV" OR "coronavirus" OR "SARSCoV-2" OR "Wuhan coronavirus" OR "Novel coronavirus" OR "2019 novel coronavirus" OR "coronavirus disease 2019 virus" OR "COVID-19 virus" OR "2019nCoV infection" OR "coronavirus disease-19."

\section{Eligibility criteria}

After collecting the articles, their title and abstract were evaluated and then, all eligible studies related to our aims and with sufficient data were included in the 
present study. For exclusion criteria, we excluded (1) posters, (2) review articles, (3) case reports, (4) letters to the editor, (5) the articles with not comprehensive data, and (6) not available article.

\section{Data collection}

After the first screening of the articles in accordance with the above-mentioned keywords in their title and abstract, the second screening was performed for finding the relevant full-text articles based on the inclusion and exclusion criteria and finally, the eligible articles were selected. All the screening process was performed independently by two reviewers (BF and HHA).

\section{Results}

\section{Literature search and screening}

Seven hundred sixty-two articles were found by a comprehensive searching on the above-mentioned electronic databases and 164 duplicated articles were removed. Five hundred ninety-eight articles were included for the first screening, 502 articles were omitted, and 96 articles were screened in their full texts. In the final step, 15 articles were eligible to be included in present study which had adequate data on chest CT findings of COVID-19-infected patients, were in English language, and contained RT-PCRconfirmed cases. The procedure of the literature searching and screening method are shown in Fig. 1.

\section{Data extraction}

The data of each article including author name, number of patients, and chest CT findings of pediatric and adult patients infected with COVID-19 were extracted and listed in Table 1.

\section{Chest CT findings of pediatric patients with COVID-19 infection}

In three studies [17-19] which reported CT features in pediatric patients infected with COVID-19 (aged 15 or younger), one also included adult patients.

Ground glass opacities (GGO) frequency reported as $60 \%$ [17], 60\% [18], and 25\% [19]. Consolidation frequency reported to be $0 \%$ [17] and $50 \%$ [19], but only one study reported mixed GGO and consolidation as $0 \%$ [17]. Pleural effusion in children reported as $0 \%$ [18] and 25\% [19] and normal imaging was seen in 20\% [18] and 25\% [19] of cases.

Pooling data from all studies, GGO was found in $55.2 \%$ of pediatric patients, consolidation in $22.2 \%$, normal imaging in 20.8\%, and pleural effusion in $4.2 \%$ of cases. Lymphadenopathy frequency was reported in a study by Liu et al. [19] as $0 \%$. Xia et al. reported halo sign in 50\% of pediatric age group, nodular opacities in $15 \%$, crazy paving in $20 \%$, and peripheral distribution (subpleural lesions) in 100\% of cases [18].

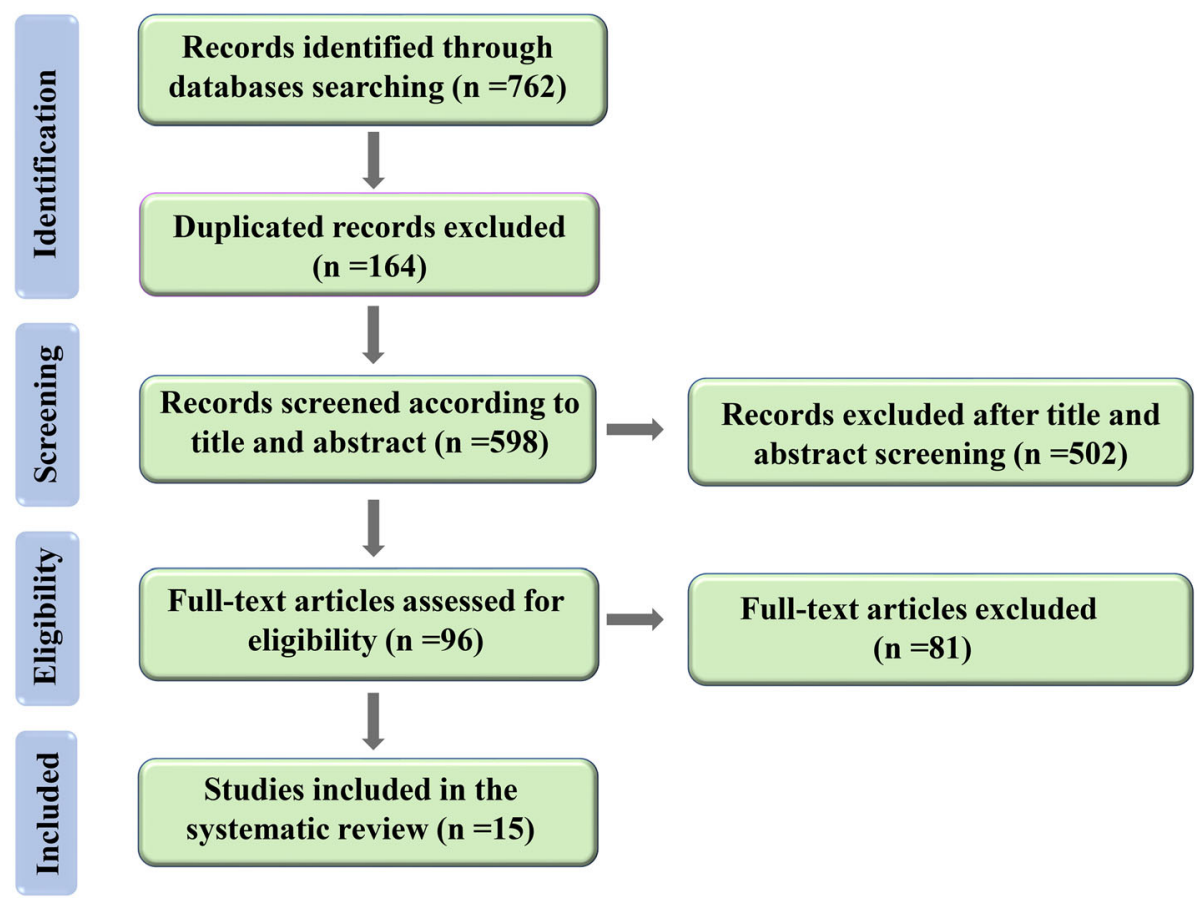

Fig. 1 PRISMA flowchart used for the study selection 


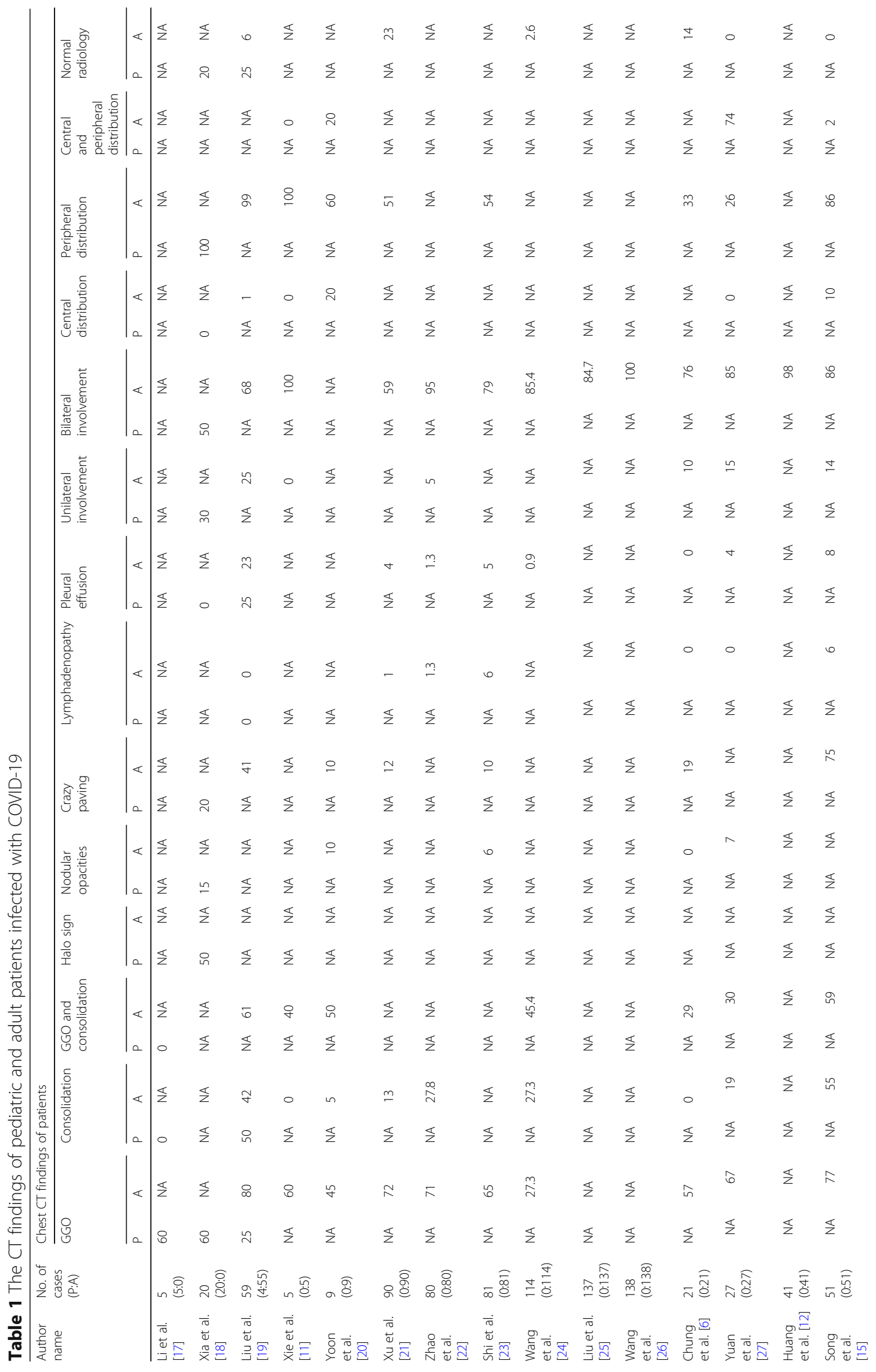




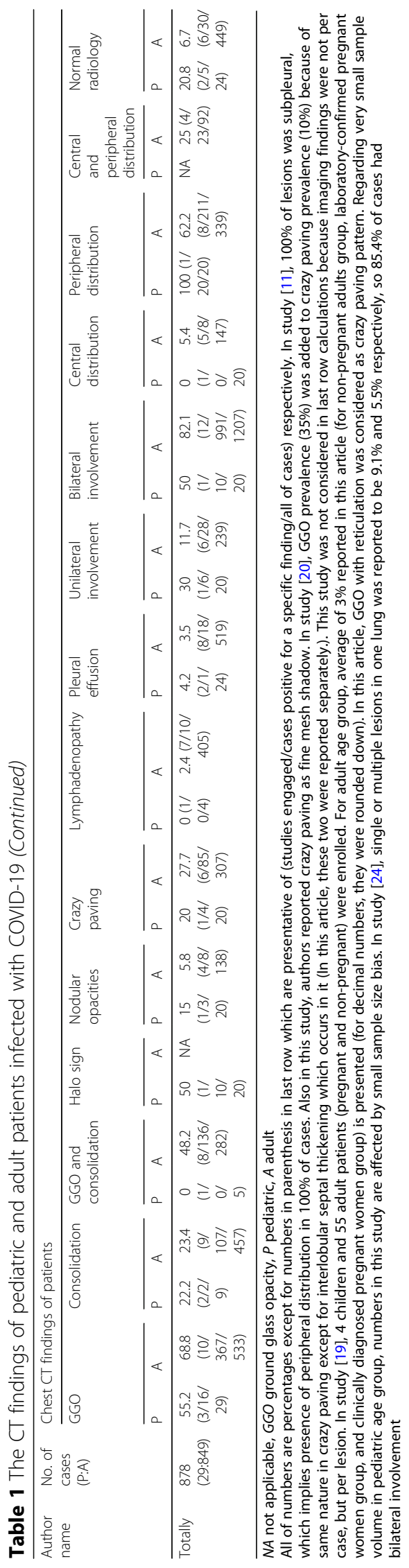




\section{Chest CT findings of adult patients with COVID-19 infection}

GGO, consolidation, and mixed density frequency were in a wide range in literature $(27.3$ to $80 \%$ [6, 10, 15, 19$24,27], 0$ to $55 \%[6,10,15,19-22,24,27]$, and 29 to $61 \%[6,10,15,19,20,24,27]$ of cases, respectively). Also, considerable diversity in reported frequencies of nodular opacities and crazy paving pattern were noted (0 to $10 \%[6,20,23,27]$ and 10 to $75 \%[6,15,19-21$, $23]$, respectively). Lymphadenopathy (0 to $6 \%[6,15,19$, $21-23,27])$, pleural effusion (0 to $23 \%[6,15,19,21-24$, $27]$ ), unilateral involvement (0 to $25 \%[6,10,15,19,22$, $27]$ ), central distribution (0 to $20 \%[6,10,15,19,22$, $27]$ ), and normal imaging (0 to $23 \%[6,15,19,21,22$, 27]) were less frequent features, as opposed to bilateral involvement (59 to $100 \%[6,10,12,15,19,21-27])$ and peripheral distribution (26 to $100 \%[6,10,15,19-21,23$, 27]) which was higher than central distribution frequency in corresponding study in all of cases, which were more encountered.

Pooling data from all studies, bilateral involvement was reported in $76.8 \%$ of adult patients, GGO in $68.4 \%$, peripheral distribution in $62.2 \%$, mixed GGO and consolidation in $48.7 \%$, consolidation in $33.7 \%$, crazy paving pattern in $27.7 \%$, mixed central and peripheral distribution in $25.0 \%$, unilateral involvement in $15.2 \%$, nodular opacities in $9.2 \%$, pleural effusion in $5.5 \%$, central distribution of lesions in $5.4 \%$, and lymphadenopathy in $2.4 \%$ of patients. A total of $9.8 \%$ of adult patients had normal imaging.

\section{Discussion}

Normal imaging was reported more frequently in infected children comparing to adults $(20.8 \%$ versus $6.7 \%$ ). Most frequent CT feature for pediatric patients was peripheral distribution and with decreasing order of frequency next features were GGO, bilateral involvement, unilateral involvement, consolidation, crazy paving pattern, nodular opacities, pleural effusion, central distribution, lymphadenopathy, and mixed consolidation and GGO (three latter features were not seen in any of investigated patients). Most frequent CT feature for adult patients was bilateral involvement and with decreasing order of frequency next features were GGO, peripheral involvement, mixed GGO and consolidation, consolidation, crazy paving pattern, mixed central and peripheral distribution, unilateral involvement, nodular opacities, pleural effusion, central distribution, and lymphadenopathy. Hereby, CT findings reported in the studies on both pediatric and adult cases enlisted above are discussed and compared (this list will not include CT features not reported in pediatric patient studies.).

\section{GGO and consolidation}

GGO is a hazy gray (slightly increased density) area without bronchovascular obscuration, causing by partial air displacement resulting from two possible mechanism, alveoli partial filling or interstitial thickening [28]. Postmortem biopsy in a COVID-19 patient showed pulmonary edema and hyaline membrane formation in both lungs [29], which can be pathologic explain of GGO [30]. Moreover, as mentioned above, there was diversity in results from different studies regarding lesion density frequency. This could be explained by symptom onset to CT scan interval. Patients with longer time interval between symptom onset and CT scan will have more consolidative lesions [15]. Lesion density gradually increased to consolidation in up to 2 weeks after disease onset in a study by Pan et al. [31], which is in consistency with a study by Shi et al. which showed that GGO can progress to or co-existed with consolidations within 1-3 weeks [23].

Consolidation reflects alveolar air occupied by fluids, cells, or tissues and manifest as density higher than GGO, high enough for bronchovascular obscuration [28]. Cellular fibromyxoid exudates in alveoli may play a role in consolidative lesions in COVID-19 [29]. For consolidation and mixed density, data available for children are from two studies with small sample size $[8,19]$ and further studies are needed to compare these CT findings in adults and children.

But generally, GGO, consolidation, and mixed GGO and consolidation all seem to occur with less prevalence in pediatric patients.

\section{Halo sign}

Halo sign is consolidative nodule or masse with peripheral ground glass opacity [28] (Fig. 2). It can be associated with viral infections and organizing pneumonia

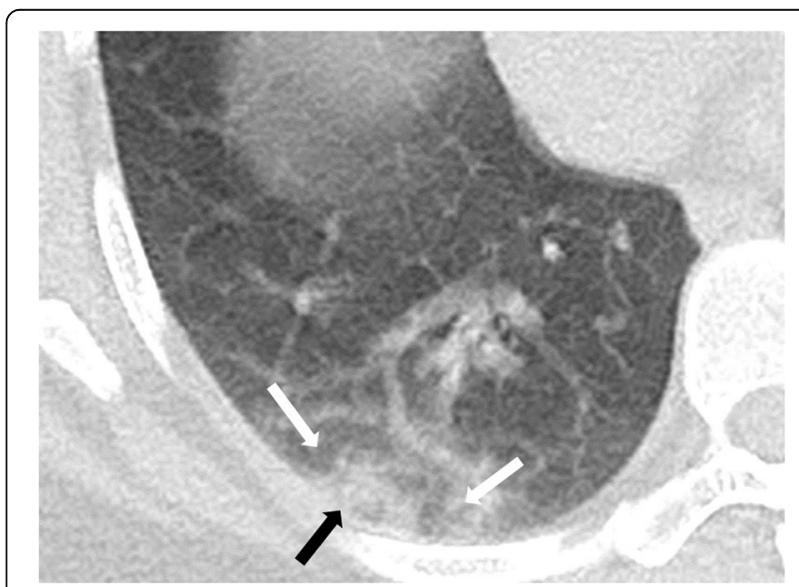

Fig. 2 Halo sign: ill-defined focus of consolidation (black arrow) is surrounded by a rim of GGO (black arrow). Various entities can give a halo sign; hence, it is rather a nonspecific sign 


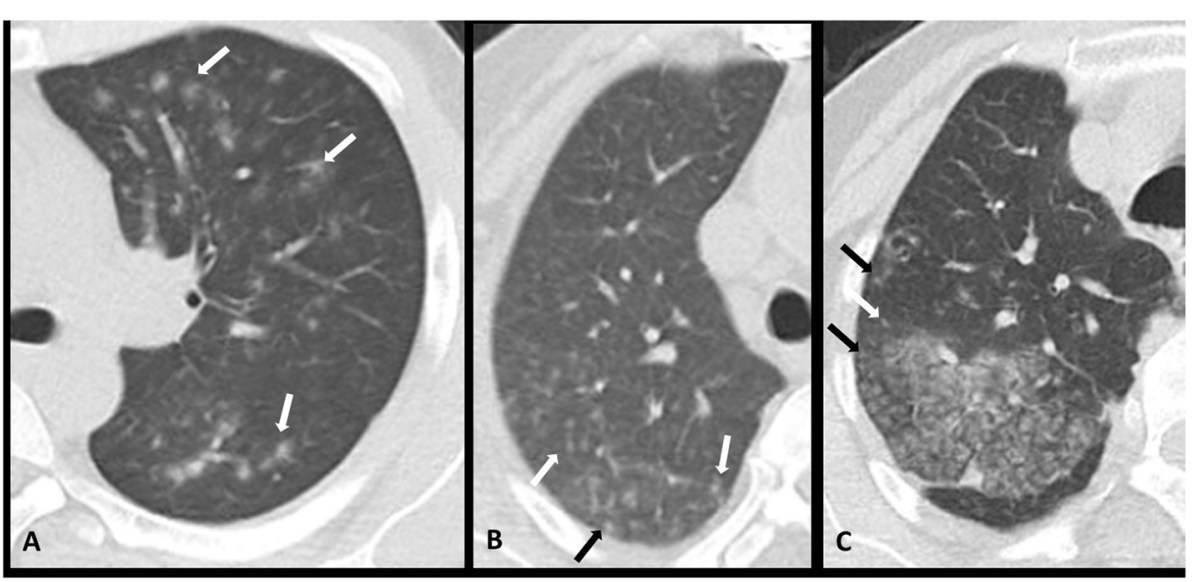

Fig. 3 Nodular opacities: small and round, ill-defined nodules of soft tissue density (some indicated with arrows) in 3 different patients with RT-PCR-confirmed COVID-19

[32]. Halo sign frequency was reported as $50 \%$ in pediatric patients [18] and is claimed to be uncommon in adults [33] and just mentioned in a case report [29] and a case series with 3 cases [34].

\section{Nodular opacities}

Nodule is defined as well or poor demarcated rounded or irregular opacity with diameter less than $3 \mathrm{~cm}$ [28] (Fig. 3) which is frequently associated with viral pneumonia [35]. Its frequency in infected children was slightly higher than adult patients (15\% versus 9.2\%). Again, only one study has reported nodular opacity frequency in children [18].

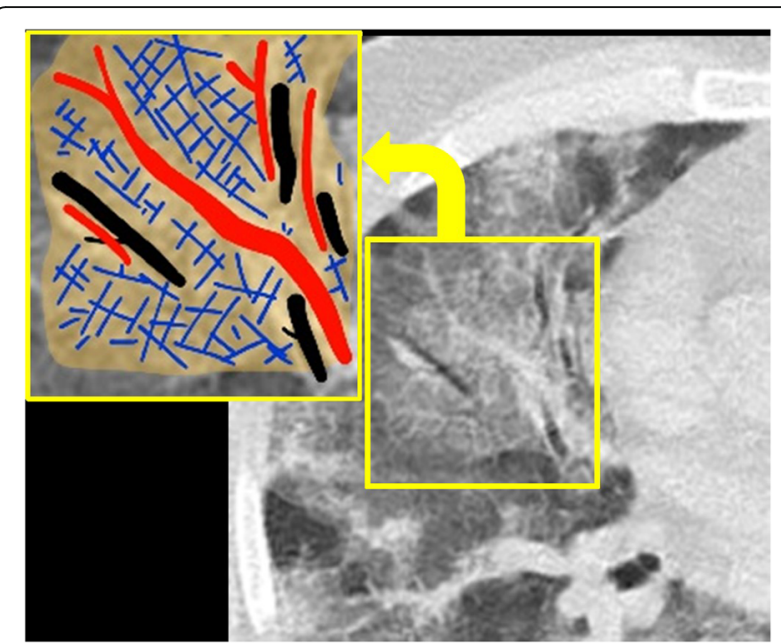

Fig. 4 Crazy paving: thickened interlobular septa (hued blue in enhanced insert) on a GGO background (highlighted in yellow) creates similar appearance to a pavement made up of disorganized paving stones. Colored in black and red are air bronchogram and pulmonary vessels

\section{Septal thickening and crazy paving}

Reticular opacities (interlobular and intralobular septal thickening) might be at least partly due to interstitial lymphocyte infiltration [29]. Reticular opacity pattern frequency increases in later stage of COVID-19 patients [23]. To our knowledge, no paper has investigated reticular pattern in children, but in adults it has been reported in some studies as 33\% in Shi et al. study [23] and $67 \%$ in Song et al. study [15].

If reticular opacity superimposes on GGO background, it looks like paving stones hence so called crazy paving pattern [28] (Fig. 4). Pathologic explain for this can be alveolar edema and interstitial inflammation due to acute lung injury $[11,36]$. It can herald infection entering progressive or peak stage [31]. Crazy paving prevalence is reported as $20 \%$ in children [18] which is slightly less frequent than that of adult patients (27.7\%). More studies with larger sample size are needed for children to provide a more presentative frequency. Also, studies on adults which take time interval between symptom onset and CT scan into consideration will troubleshoot diversities in reported prevalence.

\section{Lymphadenopathy}

Short axis diameter of $1 \mathrm{~cm}$ for mediastinal nodes is considered as thresholds discriminating enlarged nodes (lymphadenopathy based on size criterion) from normal size nodes [28]. Lymphadenopathy is one of significant risk factors of severe/critical COVID-19 pneumonia [37]. If lymphadenopathy coincides with pleural effusion and numerous lung nodules, it may suggest bacterial superinfection [13]. Lymphadenopathy is rare in both children and adults, but only one study on pediatric patients reported it with 4 cases, and none of them had lymphadenopathy [19]. 


\section{Pleural effusion}

Pleural effusion is less frequent comparing to pleural thickening and occurred in 5\% of adult cases in Shi et al. study [23]. Pleural effusion can be an indicator of poor prognosis in NCIP [23]. An autopsy report by $\mathrm{Xi}$ Liu et al. showed pleural thickening and extensive adhesion in an adult patient with COVID-19 which is what that is seen as pleural thickening with or without visible pleural effusion in CT scan [38]. Pleural effusion was rare in both children and adults $(4.2 \%$ and $3.5 \%$ respectively).

\section{Bilateral involvement}

Unlike SARS and MERS, where initial chest imaging abnormalities are more frequently unilateral, COVID19 is more likely to involve both lungs on initial imaging [39]. In a systematic review by Salehi et al., bilateral involvement in 919 cases was reported as 87.5\% [33]. Although bilateral involvement was more frequent in both adults and pediatric patients, but unilateral involvement was more frequent in children comparing to adults (30\% versus 11.7\%). Lieu et al. reported bilateral involvement in 20\% (1/5) of pediatric patients, which again emphasizes on trend toward unilaterality of lesions in pediatric patients [40] $\{\mathrm{Liu}, 2020 \# 2\}$.

\section{Axial distribution}

In several studies, it has been stated that peripheral (subpleural) distribution is typical for NCIP (Fig. 5). Salehi et al. reported $76 \%$ peripheral distribution in 121 patients (enrolled in 12 studies) [33]. In children, peripheral distribution frequency was $100 \%$ (yet more prevalent than infected adults), although this difference can be under influence of relatively small sample size in only study which reported it [18].
Vascular enlargement, reticular opacities, air-bubble sign (which was described in one study by Heng et al. [30]) or lucencies and cavities in opacified areas, air bronchogram, reverse halo (atoll) sign, craniocaudal distribution (upper/middle or lower zone involvement), and one to five lung lobes' involvement frequency (and frequency of two or more lobes' involvement) are features investigated in several adult studies, but no pediatric patients' study has been done to assess this finding prevalence yet; hence, as an interesting issue in future studies, assessment of the above-mentioned CT features in pediatric patients is suggested. This study has its limitations to be noted. First, number and sample size of pediatric patient studies was small and this can influence on imaging features' prevalence; hence, we suggest more studies with larger sample sizes to be conducted on pediatric age group with NCIP for one can compare them with infected adults' chest imaging features. Second, time gap between symptom onset and CT can influence on prevalence of findings mentioned above, regarding any CT feature time course. Categorizing CT findings in predetermined time line stages would provide more reliable frequencies and comparisons.

\section{Conclusion}

In conclusion, children infected with COVID-19 can present with normal or atypical findings (nodular opacities/unilateral involvement) in chest imaging more than adult patients; therefore, more caution should be taken to avoid misdiagnosis or missed diagnosis in infected children, and clinical and laboratory findings need to be considered more decision-making for pediatric cases with normal or atypical chest CT scan but highly suspicious of COVID-19.

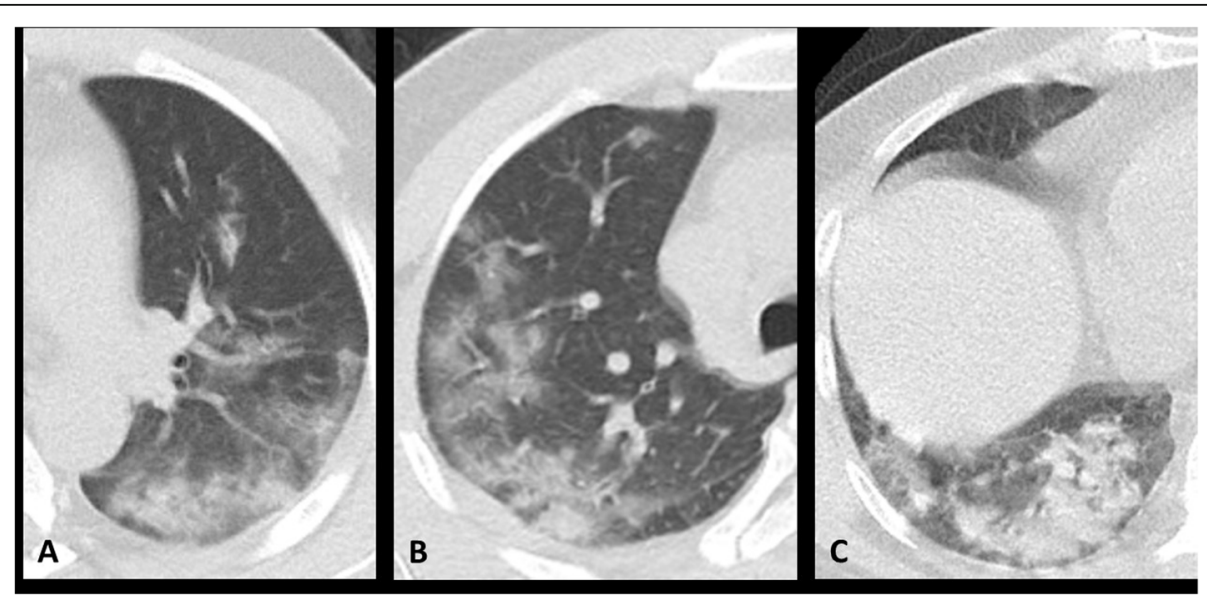

Fig. 5 Peripheral distribution: COVID-19-related pneumonia is presented as peripheral lesions in most of cases. Peripheral distribution is even more encountered in pediatric patients 


\section{Abbreviations}

CT: Computed tomography; GGO: Ground glass opacities; 2019-nCoV: 2019 novel coronavirus; NCIP: Novel coronavirus-infected pneumonia; RTPCR: Reverse transcription-polymerase chain reaction; PRISMA: Preferred Reporting Items for a Systematic Review and Meta-analysis

\section{Acknowledgements}

None.

\section{Authors' contributions}

J. A. wrote the main manuscript and analyzed the data. H. HA. performed the literature review. B.F. designed, screened, and edited the main manuscript. All authors read and approved the final manuscript.

\section{Funding}

None.

\section{Availability of data and materials}

Not applicable

\section{Ethics approval and consent to participate}

Not applicable

\section{Consent for publication}

Not applicable

\section{Competing interests}

The authors declare that they have no competing interests.

\section{Author details}

'Department of Radiology, Faculty of Medicine, Kashan University of Medical Sciences, Kashan, Iran. ${ }^{2}$ Pharmaceutical Sciences Research Center, Ardabil University of Medical Sciences, Ardabil, Iran. ${ }^{3}$ Department of Medical Physics and Radiology, Faculty of Paramedical Sciences, Kashan University of Medical Sciences, Kashan, Iran.

Received: 12 May 2020 Accepted: 22 July 2020

Published online: 04 August 2020

\section{References}

1. Li Q, Guan X, Wu P, Wang X, Zhou L, Tong Y et al (2020) Early transmission dynamics in Wuhan, China, of novel coronavirus-infected pneumonia. N Engl J Med 382:1199-1207

2. Su S, Wong G, Shi W, Liu J, Lai ACK, Zhou J et al (2016) Epidemiology, genetic recombination, and pathogenesis of coronaviruses. Trends Microbiol 24:490-502

3. Cui J, Li F, Shi ZL (2019) Origin and evolution of pathogenic coronaviruses. Nat Rev Microbiol 17:181-192

4. Wong G, Liu W, Liu Y, Zhou B, Bi Y, Gao GF (2015) MERS, SARS, and Ebola: the role of super-spreaders in infectious disease. Cell Host Microbe 18:398401

5. Novel Coronavirus-China. World Health Organization. https://www.who.int/ csr/don/12-january-2020-novel-coronavirus-china/en/. Published January 12, 2020

6. Chung M, Bernheim A (2020) CT imaging features of 2019 novel coronavirus (2019-nCoV). Radiology 295:202-207

7. Riou J, Althaus CL (2020) Pattern of early human-to-human transmission of Wuhan 2019 novel coronavirus (2019-nCoV), December 2019 to January 2020. Euro Surveill 25. https://doi.org/10.2807/1560-7917.ES.2020.25.4. 2000058

8. Hui DS, E IA, Madani TA, Ntoumi F, Kock R, Dar O, et al (2020) The continuing 2019-nCoV epidemic threat of novel coronaviruses to global health - the latest 2019 novel coronavirus outbreak in Wuhan, China. Int J Infect Dis 91:264-266

9. Zhao W, Zhong Z, Xie X, Yu Q, Liu J (2020) Relation between chest CT findings and clinical conditions of coronavirus disease (COVID-19) pneumonia: a multicenter study. AJR Am J Roentgenol 214:1072-1077

10. Xie X, Zhong Z, Zhao W, Zheng C, Wang F, Liu J (2020) Chest CT for typical 2019-nCoV pneumonia: relationship to negative RT-PCR testing. Radiology 200343
11. Wu J, Wu X, Zeng W, Guo D, Fang Z, Chen L et al (2020) Chest $C T$ findings in patients with coronavirus disease 2019 and its relationship with clinical features. Investig Radiol 55:257-261

12. Huang C, Wang Y, Li X, Ren L, Zhao J, Hu Y et al (2020) Clinical features of patients infected with 2019 novel coronavirus in Wuhan, China. Lancet 395: 497-506

13. Kanne JP, Little BP, Chung JH, Elicker BM, Ketai LH (2020) Essentials for radiologists on COVID-19: an update-radiology scientific expert panel. Radiology 200527

14. Fang $Y$, Zhang $H, X$ U Y, Xie J, Pang P, Ji W (2020) CT manifestations of two cases of 2019 novel coronavirus (2019-nCoV) pneumonia. Radiology 295: 208-209

15. Song F, Shi N, Shan F, Zhang Z, Shen J, Lu H et al (2020) Emerging 2019 novel coronavirus (2019-nCoV) pneumonia. Radiology 295:210-217

16. Moher D, Liberati A, Tetzlaff J, Altman DG (2009) Preferred reporting items for systematic reviews and meta-analyses: the PRISMA statement. Ann Intern Med 151:264-269

17. Li W, Cui H, Li K, Fang Y, Li S (2020) Chest computed tomography in children with COVID-19 respiratory infection. Pediatr Radiol. https://doi.org/ 10.1007/s00247-020-04656-7

18. Xia W, Shao J, Guo Y, Peng X, Li Z, Hu D (2020) Clinical and CT features in pediatric patients with COVID-19 infection: different points from adults. Pediatr Pulmonol 55:1169-1174

19. Liu H, Liu F, Li J, Zhang T, Wang D, Lan W (2020) Clinical and CT imaging features of the COVID-19 pneumonia: focus on pregnant women and children. J Inf Secur 80:7-13

20. Yoon SH, Lee KH, Kim JY, Lee YK, Ko H, Kim KH et al (2020) Chest radiographic and ct findings of the 2019 novel coronavirus disease (COVID19): analysis of nine patients treated in Korea. Korean J Radiol 21:494-500

21. Xu X, Yu C, Qu J, Zhang L, Jiang S, Huang D et al (2020) Imaging and clinical features of patients with 2019 novel coronavirus SARS-CoV-2. Eur J Nucl Med Mol Imaging 47:1275-1280

22. Zhao X, Liu B, Yu Y, Wang X, Du Y, Gu J et al (2020) The characteristics and clinical value of chest $C T$ images of novel coronavirus pneumonia. Clin Radiol 75:335-340

23. Shi H, Han X, Jiang N, Cao Y, Alwalid O, Gu J et al (2020) Radiological findings from 81 patients with COVID-19 pneumonia in Wuhan, China: a descriptive study. Lancet Infect Dis 20:425-434

24. Wang K, Kang S, Tian R, Zhang X, Wang Y (2020) Imaging manifestations and diagnostic value of chest CT of coronavirus disease 2019 (COVID-19) in the Xiaogan area. Clin Radiol 75:341-347

25. Liu K, Fang Y-Y, Deng Y, Liu W, Wang M-F, Ma J-P et al (2020) Clinica characteristics of novel coronavirus cases in tertiary hospitals in Hubei Province. Chin Med J. https://doi.org/10.1097/CM9.0000000000000744

26. Wang D, Hu B, Hu C, Zhu F, Liu X, Zhang J et al (2020) Clinical characteristics of 138 hospitalized patients with 2019 novel coronavirusinfected pneumonia in Wuhan. China JAMA. https://doi.org/10.1001/jama. 2020.1585

27. Yuan M, Yin W, Tao Z, Tan W, Hu Y (2020) Association of radiologic findings with mortality of patients infected with 2019 novel coronavirus in Wuhan, China. PLoS One 15:e0230548

28. Hansell DM, Bankier AA, MacMahon H, McLoud TC, Muller NL, Remy J (2008) Fleischner Society: glossary of terms for thoracic imaging. Radiology 246 : 697-722

29. Xu Z, Shi L, Wang Y, Zhang J, Huang L, Zhang C et al (2020) Pathological findings of COVID-19 associated with acute respiratory distress syndrome. Lancet Respir Med 8:420-422

30. Ye Z, Zhang Y, Wang Y, Huang Z, Song B (2020) Chest CT manifestations of new coronavirus disease 2019 (COVID-19): a pictorial review. Eur Radiol. https://doi.org/10.1007/s00330-020-06801-0

31. Pan F, Ye T, Sun P, Gui S, Liang B, Li L et al (2020) Time course of lung changes on chest CT during recovery from 2019 novel coronavirus (COVID19) pneumonia. Radiology 200370

32. Pinto PS (2004) The CT halo sign. Radiology 230:109-110

33. Salehi S, Abedi A, Balakrishnan S, Gholamrezanezhad A (2020) Coronavirus disease 2019 (COVID-19): a systematic review of imaging findings in 919 patients. AJR Am J Roentgenol:1-7. https://doi.org/10.2214/AJR.20.23034

34. Kong W. Agarwal PP (2020) Chest imaging appearance of COVID-19 infection. Radiology: Cardiothoracic Imaging 2:e200028

35. Franquet $T$ (2011) Imaging of pulmonary viral pneumonia. Radiology 260: $18-39$ 
36. Wong K, Antonio GE, Hui DS, Lee N, Yuen EH, Wu A et al (2003) Thinsection CT of severe acute respiratory syndrome: evaluation of 73 patients exposed to or with the disease. Radiology 228:395-400

37. Li X, Zeng X, Liu B, Yu Y (2020) COVID-19 infection presenting with CT halo sign. Radiology: Cardiothoracic Imaging 2:e200026

38. Xi Liu RWGQ, Wang Y et al (2020) A observational autopsy report of COVID19 (Chinese). J Forensic Med 36:19-21

39. American Roentgen Ray Society. Novel coronavirus (COVID-19) imaging features overlap with SARS and MERS: COVID-19's imaging features are variable and nonspecific, but the imaging findings reported thus far do show. ScienceDaily. ScienceDaily, 28 February 2020. Avalable from :www. sciencedaily.com/releases/2020/02/200228142018.

40. Liu M, Song Z, Xiao K (2020) High-resolution computed tomography manifestations of 5 pediatric patients with 2019 novel coronavirus. J Comput Assist Tomogr 44:311-313

\section{Publisher's Note}

Springer Nature remains neutral with regard to jurisdictional claims in published maps and institutional affiliations.

\section{Submit your manuscript to a SpringerOpen ${ }^{\circ}$ journal and benefit from:}

- Convenient online submission

- Rigorous peer review

- Open access: articles freely available online

High visibility within the field

- Retaining the copyright to your article

Submit your next manuscript at $\boldsymbol{\nabla}$ springeropen.com 